\title{
Comparative Effects Between the Commercial and Homemade Curcuma on HCT116 Adenocarcinoma Cell Line
}

\author{
Bennani Marwa ${ }^{1}$, Bruno Tiziana ${ }^{2}$, Zaïri Amira ${ }^{3}$ and Tabrelsi Mounir ${ }^{1 *}$ \\ ${ }^{1}$ Cytogenetics Laboratory, Molecular Genetics and Biology of Human Reproduction, Farhat Hached UH, Sousse, Tunisia \\ ${ }^{2}$ Department of Research, Diagnosis and Innovative Technologies, Translation research Area, Regina Elena Cancer Institute, Italy \\ ${ }^{3}$ Laboratory of Biochemistry, Faculty of Medicine Sousse, Tunisia
}

*Corresponding author: Trabelsi Mounir, Cytogenetics Laboratory, Molecular Genetics and Biology of Human Reproduction, Farhat Hached UH, Sousse, Tunisia, Laboratory of Biochemistry, Faculty of Medicine Sousse Tunisia.

To Cite This Article: Bennani Marwa, Bruno Tiziana, Zaïri Amira, Tabrelsi Mounir. Comparative Effects Between the Commercial and Homemade Curcuma on HCT116 Adenocarcinoma Cell Line. Am J Biomed Sci \& Res. 2021 - 14(2). AJBSR.MS.ID.001980. DOI: 10.34297/AJBSR.2021.14.001980.

Received: 岿July 26, 2021; Published: 畊 September 28, 2021

\begin{abstract}
Background: Curcuma longa is widely used for its ability to inhibit carcinogenesis and the capacity to exert a chemo-preventive effect in different types of cancers including colorectal cancer. While our food habits have changed, the prevalence of colorectal cancer have not stopped increasing.
\end{abstract}

Materials and methods: We investigated the anti-cancerous effect of the aqueous extract of a commercial Curcuma (cur C) compared to the traditional one (cur T) on the adenocarcinoma cell line HCT116 in order to evaluate if the increase in colorectal cancer rates is related to changes in dietary habits.

Results: Cur T exhibits a physiological activity on colon cancer, assuming its possible use in prevention and cancer therapy.

Conclusion: The change in our eating habits from traditional to commercial can be a direct cause of the increase of cancer in the world and more precisely in Tunisia.

Keywords: Curcuma, HCT 116 cells, adenocarcinoma colon cancer, anticancer treatment.

\section{Introduction}

Cancer is the major leading cause of morbidity and mortality in the world. In fact, colorectal cancer (CRC) is the most frequent type of cancer in the world and it is the second most common type of cancer in both genders (women: 10.1\%; men: 12.4\%) [1]. Several data showed that the number of newly diagnosed CRC cases continues to grow especially in the developing countries [2]. For several years, Tunisia has had a low incidence of CRC that seems very far from developed countries, but significant variation in colorectal cancer incidence rates and trends have been observed in our country, without a consistent screening program. The predicted CRC incidence rate by 2024 should be $39.3 / 100,000$ for males and
22.9/ 100,000 for females [3]. Natural products from plants have been intensively explored to find compounds providing against a large number of diseases associated to cancer. The Mediterranean kitchen is one of the most known, and contains a unique mixture of flavors, tastes and nutrients that have no equal around the world [4]. The Tunisian kitchen is a good example. Actually, the Romans and Carthaginians, and well before them the Greeks, brought a lot of recipes and left for us a huge traditional food heritage. This kitchen is reputed for its proper combination that has never been elaborated. It is characterized by a special use of herbs and aromatic plants [5], and Curcuma is one of these spices. It is widely 
used in our traditional dishes [6] and it seems to have anticancer activity due to its active ingredient: the Curcumin, which is present in its rhizome and rootstock [7]. Previous studies have shown that Curcumin has an inhibitory action in all phases of cancer growth, which are initiation, promotion, and propagation [8]. However, some studies report that many traditional dishes using certain spices became absent from the table of many families, and even forgotten over time [9]. We have also noted that Tunisians use more frequently the commercial Curcuma instead of mashing the dried roots as did our ancestors. Moreover, the incidence of colorectal cancer in Tunisia is increasing and it is important for us to know if we have also lost the preventive power of our kitchen. Would the change in the origin of these spices be associated with the increase in cancer?

Thus, the aim of this study was to evaluate the association between the origin of spices that we use in our diet and the prevalence of CRC. In this context, we tried to describe the effects of such diet on the incidence of CRC and we aimed to elucidate the comparative effects between the commercial curcuma and the homemade one on HCT116 adenocarcinoma cell line.

\section{Materials and Methods}

\section{Cell Culture}

Human colorectal carcinoma cell lines (HCT116) were cultured in DMEM medium containing 10\% heat- inactivated fetal bovine serum, a mixture of penicillin $(100 \mathrm{U} / \mathrm{ml})$ and streptomycin $(100$ $\mu \mathrm{g} / \mathrm{ml}$ ) and incubated at $37{ }^{\circ} \mathrm{C}$ in a humidified atmosphere with $5 \%$ CO2. The cell numbers were counted by a hematocytometer, and the viability was always greater that $95 \%$ in all experiments as assayed by the $0.025 \%$ trypan blue exclusion method. Further dilutions of stock solutions of curcuma were made in culture media just before use. In all experiments, the final concentration of DMSO did not exceed $0.3 \%(\mathrm{v} / \mathrm{v})$, a concentration which is non-toxic to the cells. The same concentration was present in control experiments.

\section{Plant Material}

We bought turmeric in two forms, the first was dry rhizomes, and the second was the powder of a local market. We referred to the recipes of our grandmothers and their home-made spice preparations. We milled the rhizomes to have fine powder without any additives. In this way, we had the traditional Curcuma (cur T), and the purchased powder was defined as the commercial Curcuma ( $\operatorname{cur}$ C). We also used Curcumine from Sigma Aldrich as a positive control.

\section{Preparation of Extracts}

Decoctions were performed by adding $200 \mathrm{ml}$ of distilled water to the sample $(1 \mathrm{~g})$, heated and boiled for $5 \mathrm{~min}$. Then, the mixture was filtered under reduced pressure then frozen and lyophilized. The obtained filtrate was deeply frozen at $-70^{\circ} \mathrm{C}$ and lyophilized (freeze dried) using lyophilizer to collect crude aqueous extract. For the treatment, turmeric was dissolved in the culture medium.

\section{DPPH Radical Scavenging Assay}

The antioxidant activity of extracts was performed using the stable DDPH (1,1-diphenyl-2picrylhydrazyl radical) according to the method reported by Kartal $N$ et al [10]. A volume of 180 $\mu \mathrm{l}$ of various concentrations of extracts $(0,009-0,312 \mu \mathrm{g} / \mathrm{ml})$ was added to $1620 \mu \mathrm{l}$ of DDPH. The absorbance was observed at $515 \mathrm{~nm}$ and correlated to the ability of extract to reduce the stable radical DPPH to the yellow-colored diphenylpicrylhydrazine. The antiradical activity was expressed as $\mathrm{IC}_{50}$, and corresponded to the extract dose required to induce $50 \%$ inhibition. The lower $\mathrm{IC}_{50}$ value corresponds to a higher antioxidant activity of plant extract. The ability to scavenge the DPPH radical was calculated using the following equation: Scavenging activity $(\%)=[(\mathrm{A} 0-\mathrm{A} 1)] / \mathrm{A} 0 * 100$

\section{Phase Contrast Microscopy}

Cell morphology and cell density was observed after $48 \mathrm{H}$ of treatment with cur $T$ or cur $C$ under an inverted-phase contrast microscope (Axiovert 40 CFL,Zeiss).

\section{Anti-proliferative activity assay}

The cytotoxicity of samples in HCT116 cells was analyzed by colorimetric 3-(4,5-dimethyl-2-thiazolyl-)-2,5-diphenyl2Htetrazolium bromide (MTT assay). Briefly, $1 \times 104$ exponentially growing cells were seeded in 96-well micro-culture plates with various samples concentrations. After being incubated for $24 \mathrm{~h}$, the cells were treated with different concentrations of samples for $24 \mathrm{~h}$. After the addition of MTT $(0.5 \mathrm{mg} / \mathrm{ml})$, cells were incubated at $37^{\circ} \mathrm{C}$ for $4 \mathrm{~h}$. Cells for negative control were treated only with vehicle dimethyl sulfoxide (DMSO).After $4 \mathrm{~h}$ incubation, the culture medium was discarded by gentle aspiration and replaced by $200 \mu \mathrm{L}$ of DMSO to dissolve the formazan crystals. The amount of formazan product was measured at $570 \mathrm{~nm}$ and $650 \mathrm{~nm}$ as a background using a microplate reader. The number of viable cells is corresponding to the production of formazan. Cell viability was calculated and expressed as percentages according to the following equation: Cell viability $(\%)=[$ Sample $/$ Control $] \times 100$

\section{Nuclear Morphology Detection using Hoechst 33342}

HCT116 cells $(5 \times 104$ cells $)$ were plated in $60 \mathrm{~mm}^{2}$ culture dishes and incubated in $5 \% \mathrm{CO}_{2}$ incubator at $37{ }^{\circ} \mathrm{C}$ for $24 \mathrm{~h}$. After incubation, the cells were treated with different concentrations of samples while negative control was treated with vehicle DMSO for $48 \mathrm{~h}$. The cells were then harvested and washed with PBS and subsequently stained with Hoechst $33342(40 \mu \mathrm{g} / \mathrm{mL})$ at room 
temperature in the dark for $30 \mathrm{~min}$. Then, the cells were observed under inverted fluorescence microscope. For each sample, 300 cells were examined.

\section{Western Blot}

Cell extracts purifications and Western blotting analyses were performed as previously described (Desantis etal., 2015) using the following antibodies: p53 (D07) (Santacruz Biotechnology: sc47698), PUMA(B-6) (Santacruz Biotechnology: sc-377015), p85 (Millipore ABS-234). Secondary used antibodies were goat antimouse (Biorab 1706516) and goat anti-rabbit (Biorad 1706515), conjugated to horseradish peroxidase. $\alpha$ - $\beta$-actin actin (Sigma Aldrich, \#A5441). Immuno-stained bands were detected by the chemiluminescent method (Pierce RPN2232).

\section{LDH-Cytotoxicity Assay}

Cells were seeded in 96-well culture plates ( 5 x 104 cells/well). After being incubated for $24 \mathrm{~h}$, cells were treated with different concentrations of samples for $48 \mathrm{~h}$. Lactate dehydrogenase (LDH) activity was determined using the LDH-Cytotoxicity Colorimetric Assay Kit from BioVision (Milpitas, CA, USA) following the manufacturer's protocol. The absorbance of LDH in the culture medium was measured at $340 \mathrm{~nm}$ as a background using a microplate.

\subsection{Statistical Analysis}

SPSS Version 16 was used to perform the statistical analysis. Testing of parameters was carried-out using Student's t-test and one-way variance analysis. $\mathrm{p}<0.05$ was considered statistically significant.

\section{Results}

\section{Comparison of the Antioxidant Activity}

All tested extracts showed a promising DPPH scavenging effect in a concentration-dependent manner. The lower IC $_{50}$ corresponds to the highest radical scavenging effectiveness. This was observed with $\operatorname{cur} T(3,45 \pm 0,1)$. This result highlights the greater antioxidant activity of the cur $T$ than $\operatorname{cur} C$. The results of the DPPH scavenging activity of $\operatorname{cur} T$ and $\operatorname{cur} C$ are shown in Table1.

Table 1: $\mathrm{IC}_{50}$ values of the extracts of cur T and cur $\mathrm{C}$ in DPPH assay. Data are means $\pm S D$

\begin{tabular}{|l|r|}
\hline \multicolumn{2}{|c|}{ DPPH IC $_{\mathbf{5 0}}$ Scavenging $(\boldsymbol{\mu g} / \mathbf{m l})$} \\
\hline Cur $T$ & $3,45 \pm 0,010$ \\
\hline Cur $C$ & $11,5 \pm 0,015$ \\
\hline
\end{tabular}

$\mathrm{IC}_{50}$ values correspond to the sample concentration providing $50 \%$ of antioxidant activity. Data are means \pm SD of the two independent extract.

\section{Cytotoxicity effect of cur $\mathrm{T}$ and cur $\mathrm{C}$ on the morphology of HCT116 cell line}

After the treatment of HCT116 with cur $C$ and cur $T$ we observed a plasma membrane blebbing, cell shrinkage and cell detachment only in the sample treated with cur T. It clearly showed the apoptotic mechanisms that started through the altered cell membranes (Figure 1).

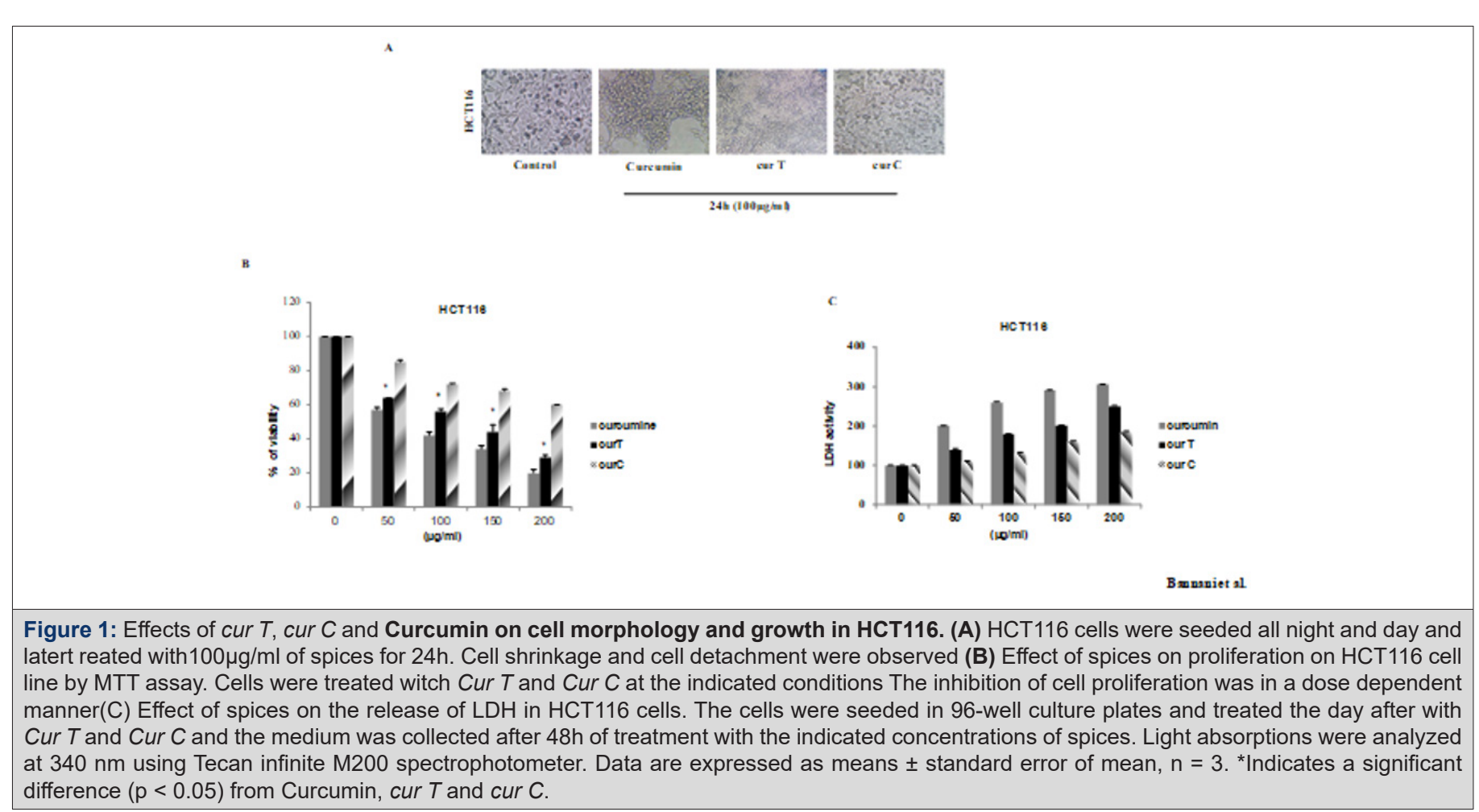


To investigate the potential inhibitory activity of these compounds on HCT116 cells proliferation, we used the MTT assay. As shown in (Figure1B), both treatments with cur T and cur $C$ were able to inhibit cell proliferation in a dose-and time-dependent manner, but the results also show a higher effect of the cur $T$ compared to the $\operatorname{cur} C$ because, at the lowest doses of $\operatorname{cur} T$, strong cytotoxicity is achieved. To further confirm this cytotoxic effect, we performed a colorimetric assay to quantitatively measure lactate dehydrogenase (LDH) released into the media from damaged cells as a biomarker of cytotoxicity and cytolysis. This assay was originally used to quantify cell death occurring via necrosis but has been also shown to accurately measure apoptosis. As we can see in (Figure 1C), there was an increase of LDH presence in the culture medium of cells treated with cur $T$ at all used doses, while a similar, but lower effect was obtained with the cur $C$. These results further demonstrated the best activity of cur TinHCT116 adenocarcinoma cell line.

\section{Cur T Induces Apoptosis}

The staining by Hoechst 33342 exhibited numerous cells with blebbing, condensation and DNA fragmentation, after the treatment with cur $\mathrm{T}$ and $\operatorname{cur} C$ for $48 \mathrm{H}$, but no significant nuclear fragmentation in the control group was observed (Figure 2A). These results demonstrate that curcuma induces apoptosis but the best results were obtained through the treatment of cells by cur $T$. These differences are clearly noticeable in the graph in (Figure2B). As a further demonstration of the major apoptotic effect of cur T in the studied cell line we performed a FACS analysis after treatment with both spices. As a result, the treatment with cur Thas shown a greater quantity of cells in the sub G1 phase, characteristic of apoptosis, even with the fewest concentration $(25 \mu \mathrm{g} / \mathrm{ml}$ ) (Figure 2C).

Based on the observation by FACS analysis, we investigated whether these compounds were capable of regulating some of the key players of cell cycle progression. After treatment with cur T and $\operatorname{cur} C$, we evaluated the expression of proteins which are usually involved in apoptosis and in DNA damage response (Figure 2D). The most important alteration in human tumurogenesis is the deletion or the alteration of $\mathrm{p} 53$ gene which is a tumor suppressor. This gene can be activated by several stress condition or DNA damage. As shown in (Figure 2D), treatment with Cur T significantly increase in a dose dependent manner. The induction of p53 started as early with $50 \mu \mathrm{g} / \mathrm{ml}$ and reached the highest level with $100 \mu \mathrm{g} / \mathrm{ml}$. In addition, we have observed an increase of the level of the apoptosis-inducing factor PUMA (with $100 \mu \mathrm{g} / \mathrm{ml}$ ) which is an essential mediator of the apoptotic function of p53. Finally, we demonstrated an increase of p85 PARP fragment that is the result of the caspase- 3 cleavage protein typical marker of apoptosis.
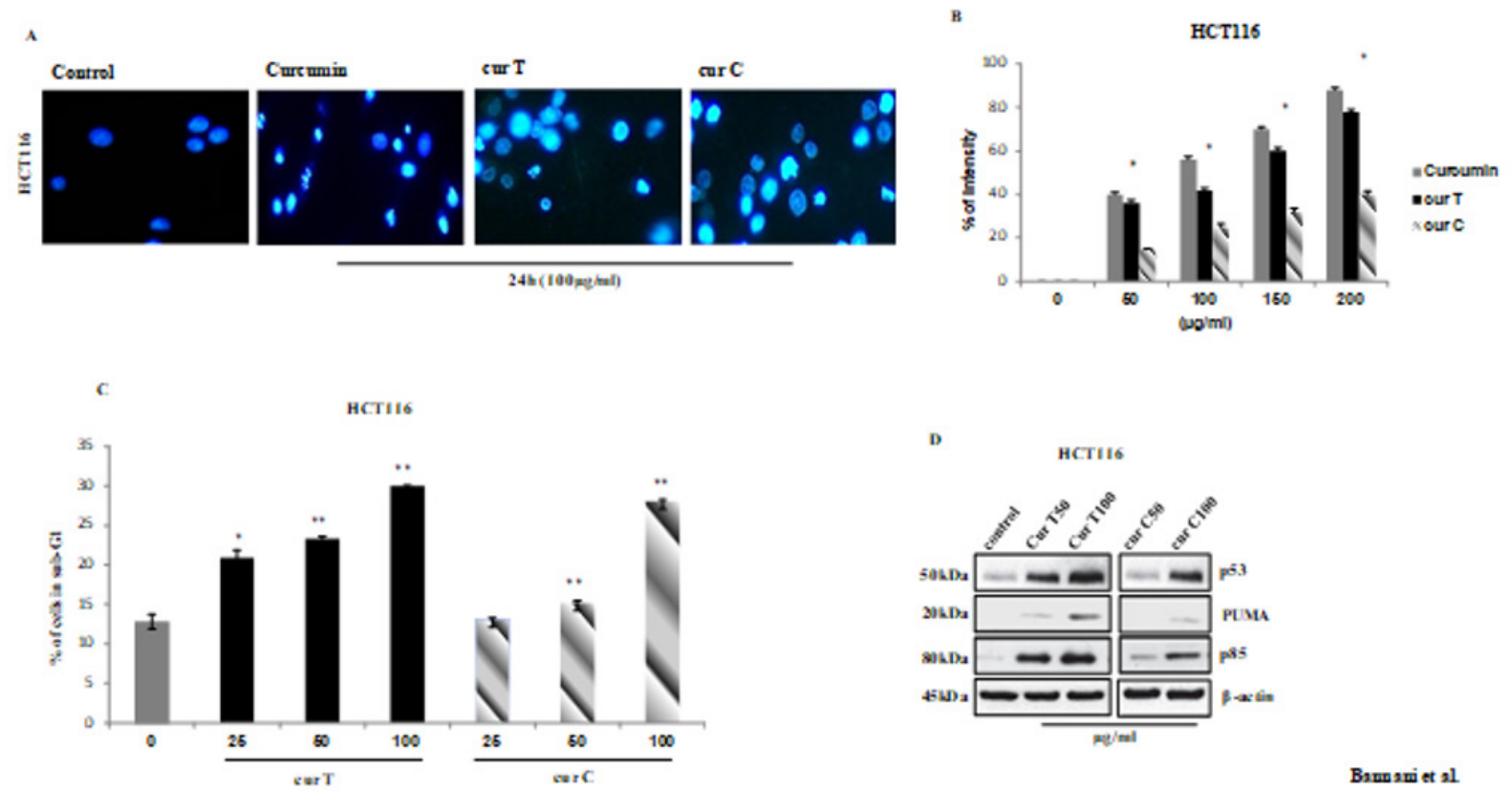

Figure 2: Induction of apoptosis in HCT116 cells by spices treatment.(A) Nuclei of HCT116 cells have been stained with fluorescent DNA-binding dye (Hoechst 33342)and analyzed under fluorescence microscopy using automated analytical software. The detected intensity is represented in the graph. (B) Data are expressed as means \pm standard error of mean, *Indicates a significant difference ( $p<0.05)$ from control. cur $T$, cur $C$ and Curcumin. The DNA contents of the cells after the treatment with spices for $24 \mathrm{~h}$ were evaluated by FACS analysis using propidium iodide staining. Representative data from the cell cycle analyses are shown. The percentage of sub-G1 phase cells from three different FACS analyses were calculated and presented as the mean \pm standard error of the mean. ${ }^{*} P<0.05$. (C) Equal amounts of the proteins extracted from SCE treated cells were subjected to western blot analysis for detection of apoptotic signaling molecules, including p53, PUMA and p85. (D) 


\section{Discussion}

Numerous studies have shown that there is a correlation between regular consumption of fruits and vegetables and prevention of developing lifestyle disorders, such as cancer [11]. Diet and nutrient factors are widely believed to act as pro- and antitumor risk modifiers across the entire multistep process of colorectal tumorigenesis [12]. Curcuma longa seems to be an antiproliferative agent and seems to possess many of the desirable qualities for preventing cancer and could have great potential as chemopreventive [13].

The present research study was directed to check if the change of spices used in our kitchen is related to the increase of CRC cancer rate, that's why we compared the antioxidant and anti-proliferative effects of traditional homemade Curcuma (cur T) and commercial Curcuma ( cur C) on colorectal cells HCT116.

Our results show that cur $T$ display the strongest DDPH (2,2-diphenyl-1-picrylhydrazyl) radical-scavenging activity comparing it to $\operatorname{cur} C$. The lower $\mathrm{IC}_{50}$ corresponds to the highest radical scavenging effectiveness observed with cur $T$. A lot of studies showed an important antioxidant effect of curcuma [14] and others concluded that this effect is due to Curcumin [13] but Curcuma contains other molecules that have the same effect [15]. Indeed, in our study, cur T showed a promising DPPH scavenging effect in a concentration-dependent manner. These results provide convincing proof of the protective effect of cur T treatment against HCT116, which suggests that cur Thas an anti-proliferative action in HCT116 cells and can potentially serve as an anti-proliferative agent for cancer. A few studies have also revealed that the antioxidant proprieties may directly cause the cytotoxic effect of Curcuma [14]. Indeed, other studies prove the pro-apoptotic [16] and antiproliferative effect of Curcuma and Curcumin [14], which was also proved by our results by several techniques. In order to evaluate whether our plants extracts are safe for a possible therapeutic use or not, we tested their potential in vitro cytotoxicity in confluent monolayers of human HCT116 cells using the MTT cell viability assay. Our results revealed that, even when we used the fewest dose of cur T, they induce more than $50 \%$ of mortality. According to the literature, curcuma possesses potent antitumor activity, which may be due to its direct cytotoxic effect or antioxidant properties. This test was consolidated by the LDH release test. Such assay was originally used to quantify cell death occurring via necrosis but also has been shown to accurately measure apoptosis [16] and $\operatorname{cur} T$ was cytotoxic as assessed biochemically by an increase in LDH content in the supernatant in a dose-dependent manner. We also found with Hoechst typical ultrastructural modifications such as dense and/or fragmented nuclei of cells treated by cur $T$ but no significant nuclear fragmentation in the control group was observed. In addition, the investigation of cell cycle demonstrated that the population of sub-G1 phase cells was increased by cur $T$ treatment in a dose-dependent manner as it was reported [17]. G1phase arrest correlated with the regulation of tumor protein. Tumor suppressor genes, including p53 and PUMA are the main molecular targets for several herbal drugs. Many studies reported that these genes caused arrest of cell cycle at the G0/G1 phase of human colorectal carcinoma (HCT116 cells) by up regulating the p53. It was recognized as the protein which controls G1 phase which will both induce cell arrest in G1 phase and induce apoptosis in order to maintain tissue homoeostasis [18]. Curcuma has been shown to increase the expression of these tumor suppressor $[19,20]$. Our finding demonstrated that Cur T increases the p53, p21 and PUMA protein expression more than did Cur C. As it was reported, Curcuma and its compounds could induce apoptosis of all cancer cells tested in this study in a dose-dependent manner, indicating possibility of curative and preventive windows [21]. Previous studies have evaluated the anti-oxidant and pro-apoptotic effect of Curcuma root. Our results are comparable to these studies. However, the cur $C$ effects were not previously evaluated in literature. According to our findings the cur $T$ has better effect on HCT116 cells. Culinary habits have dramatically changed with the emergence of commercially proceeded food [22]. The impact of these changes has increased the risk of CRC. The ratios of bioactive molecules are decreased in commercial food leading to the loss of preventive effect against cancer of natural nutrients. This fact is reinforced by our findings.

All taken together, the commercial Curcuma lacks much of its benefits and the use of traditional Curcuma is encouraged. Further investigations should be performed to identify all the bioactive molecules in Curcuma (besides Curcumin). This would allow the complementation of commercial food by the bioactive molecule. Commercial products must undergo controls to validate the health effects. Nevertheless, replication of these findings in other studies is required in order to confirm the reported associations and to be able to make public health recommendations.

\section{Acknowledgments}

This work is supported by grants from Ministry of higher education and scientific research of Tunisia. We would like to thank PhD. Maurizio Fanciulli and all the members of the Department of Research, Diagnosis and Innovative Technologies, Translation research Area, Regina Elena Cancer Institute in Rome for their cooperation. 


\section{References}

1. Li M, Yue Gg-L, Tsui Sk-W, Fung K-P, Lau Cb-S (2018) Turmeric extract, with absorbable curcumin, has potent anti-metastatic effect in vitro and in vivo. Phytomedicine 46: 131-141.

2. Roncucci L, Mariani F (2015) Prevention of colorectal cancer: How many tools do we have in our basket? Eur. J. Intern. Med. 26 Elsevier: 752-756.

3. Missaoui N, Jaidaine L, Abdelkader A Ben, Trabelsi A, Mokni M, et al (2011) Colorectal cancer in Central Tunisia: increasing incidence trends over a 15-year period. Asian Pacific J Cancer Prev Apjcp 12: 1073-1076.

4. Alberto Bianchi (2015) Bianchi A. The Mediterranean aromatic plants and their culinary use. Nat Prod Res 29: 201-206.

5. Zairi A, Nouir S, M`hamdi N, Bennani M, Bergaoui I, et al. (2018) Antioxidant, Antimicrobial and the Phenolic Content of Infusion, Decoction and Methanolic Extracts of Thyme and Rosmarinus Species. Curr Pharm Biotechnol 19: 590-599.

6. Hoffman R, Gerber M. (2015) Food processing and the mediterranean diet. Nutrients 7: 7925-7964.

7. Chaithongyot S, Asgar A, Senawong G, Yowapuy A, Lattmann E, et al (2015) Anticancer effects of Curcuma C20-dialdehyde against colon and cervical cancer cell lines. Asian Pacific J Cancer Prev 16: 6513-6519.

8. Fridlender M, Kapulnik Y, Koltai H. (2015) Plant derived substances with anti-cancer activity: From folklore to practice. Front. Plant Sci 6: 799.

9. Di Bella G, Potortì Ag, Ben Tekaya A, Beltifa A, Ben Mansour H, et al. (2019) Organic contamination of Italian and Tunisian culinary herbs and spices. J Environ Sci Heal - Part B Pestic Food Contam Agric Wastes 54: 345-356.

10. Kartal N, Sokmen M, Tepe B, Daferera D, Polissiou M (2007) Investigation of the antioxidant properties of Ferula orientalis L. using a suitable extraction procedure. Food Chem 100: 584-589.

11. Li Yh, Niu Yb, Sun Y, Zhang F, Liu Cx, et al. (2015) Role of phytochemicals in colorectal cancer prevention. World J Gastroenterol 21: 9262-9272.

12. Martínez-González Má, Hershey Ms, Zazpe I, Trichopoulou A (2017) Transferability of the Mediterranean diet to non-Mediterranean countries. What is and what is not the Mediterranean diet. Nutrients 9: 1-14.
13. Rajkumari S, Sanatombi K (2017) Nutritional value, phytochemical composition, and biological activities of edible Curcuma species: A review. Int J Food Prop 20: S2668-S2687.

14. Karmakar I, Haldar S, Chakraborty M, Dewanjee S, Haldar Pk (2016) In vitro antioxidant and cytotoxic activity of Zanthonitrile isolated from Zanthoxylum alatum. J Appl Pharm Sci 6: 119-122.

15. Isnaini I, Permatasari N, Mintaroem K, Prihardina B, Widodo Ma (2018) Oxidants-Antioxidants Profile in the Breast Cancer Cell Line. Asian Pac J Cancer Prev, 19: 3175-3178.

16. Yang Sm, Tsai Kd, Wong Hy, Liu Yh, Chen Tw, et al. (2016) Molecular mechanism of Cinnamomum verum component cuminaldehyde inhibits cell growth and induces cell death in human lung squamous cell carcinoma NCI-H520 cells in vitro and in vivo. J Cancer 7: 251-261.

17. Hong Gw, Hong Sl, Lee Gs, Yaacob H, Malek Sna (2016) Non-aqueous extracts of Curcuma mangga rhizomes induced cell death in human colorectal adenocarcinoma cell line (HT29) via induction of apoptosis and cell cycle arrest at G0/G1 phase. Asian Pac J Trop Med 9: 8-18.

18. Tyagi Ak, Prasad S, Yuan W, Li S, Aggarwal Bb (2015) Identification of a novel compound ( $\beta$-sesquiphellandrene) from turmeric (Curcuma longa) with anticancer potential: Comparison with curcumin. Invest New Drugs 33: 1175-1186.

19. Alkhader E, Roberts Cj, Rosli R, Yuen Kh, Seow Ek, et al. (2018) Pharmacokinetic and anti-colon cancer properties of curcumin-containing chitosan-pectinate composite nanoparticles. J Biomater Sci Polym Ed 29(18): 2281-2298.

20. Zheng J, Zhou Y, Li Y, Xu Dp, Li S, et al. (2016) Spices for prevention and treatment of cancers. Nutrients, 8(8): 495.

21. De Campos PS, Matte BF, Diel LF, Jesus LH, Bernardi L, et al. (2017) Low Doses of Curcuma longa Modulates Cell Migration and Cell-Cell Adhesion. Phyther Res 31: 1433-1440.

22. Desantis A, Bruno T, Catena V, De Nicola F, Goeman F, et al. (2015) Che-1induced inhibition of mTOR pathway enables stress-induced autophagy. EMBO J 34: 1214-1230. 